\title{
Kinetic market models with single commodity having price fluctuations
}

\author{
Arnab Chatterje\& and Bikas K. Chakrabart \\ Theoretical Condensed Matter Physics Division and Centre for Applied Mathematics and Computational Science, \\ Saha Institute of Nuclear Physics, 1/AF Bidhannagar, Kolkata 700064, India.
}

\begin{abstract}
We study here numerically the behavior of an ideal gas like model of markets having only one non-consumable commodity. We investigate the behavior of the steady-state distributions of money, commodity and total wealth, as the dynamics of trading or exchange of money and commodity proceeds, with local (in time) fluctuations in the price of the commodity. These distributions are studied in markets with agents having uniform and random saving factors. The self-organizing features in money distribution are similar to the cases without any commodity (or with consumable commodities), while the commodity distribution shows an exponential decay. The wealth distribution shows interesting behavior: Gamma like distribution for uniform saving propensity and has the same power-law tail, as that of the money distribution, for a market with agents having random saving propensity.
\end{abstract}

PACS numbers: 89.20.Hh,89.75.Hc,89.75.Da,43.38.Si

\section{INTRODUCTION}

The study of wealth distribution [1] in a society has remained an intriguing problem since Vilfredo Pareto who first observed [2] that the number of rich people with wealth $m$ decay following an inverse power-law:

$$
P(m) \sim m^{-(1+\nu)} .
$$

$P(m)$ is the number density of people possessing wealth $m$ and $\nu$ is known as the Pareto exponent. This exponent generally assumes a value between 1 and 3 in different economies and times [1, 3, 4, 5]. It is also known that for low and medium income, the number density $P(m)$ falls off much faster: exponentially [3] or in a log-normal way [4].

In recent years, easy availability of data in electronic media has helped in the analysis of wealth or income distributions in various societies [1]. It is now more or less established that the distribution has a power-law tail for the large (about $5 \%$ of the population) wealth/income while the majority (around 95\%) low income distribution fits well to Gibbs or log-normal form [1, 2, 3, 4, 5, 6].

There have been several attempts to model a simple economy with minimum trading ingredients, which involve a wealth exchange process [1] that produce a distribution of wealth similar to that observed in the real market. We are particularly interested in microscopic models of markets where the (economic) trading activity is considered as a scattering process [7, 8, 9, 10, 11, 12, 13, 14] (see also Ref. [15] for recent extensive reviews). We concentrate on models that incorporate 'saving propensity' (of the agents) as an essential ingredient in a trading process, and reproduces the salient features seen across wealth distributions in varied economies (see Ref. [16] for

\footnotetext{
*Electronic address: arnab.chatterjee@saha.ac.in
}

${ }^{\dagger}$ Electronic address: bikask.chakrabarti@saha.ac.in a review). Much earlier, Angle [17] studied an inequality process, which can be mapped to the uniform savings models is certain cases; see Ref. [18] for a detailed review.

These studies also show (and discussed briefly here) how the distribution of savings can be modified to reproduce the salient features of empirical distributions of wealth - namely the shape of the distribution for the low and middle wealth and the tunable Pareto exponent. In all these models [9, 10, 11, 12, 13], 'savings' was introduced as a quenched parameter that remained invariant with time (or trading).

Apart from presenting a brief summary in Section II (giving the established results in such models), we present new results for a similar (gas like) market model, where the exchange is for a non-consumable commodity (globally conserved, like money). We find, although the details of the steady-state money and wealth (money and commodity together) distributions differ considerably, the same Pareto tail feature appears in both, with identical exponent $(\nu)$ value.

\section{IDEAL-GAS LIKE MODELS OF TRADING MARKETS WITHOUT ANY COMMODITY}

\section{A. Without savings}

We first consider an ideal-gas model of a closed economic system. Wealth is measured in terms of the amount of money possessed by an individual. Production is not allowed i.e, total money $M$ is fixed and also there is no migration of population i.e, total number of agents $N$ is fixed, and the only economic activity is confined to trading. Each agent $i$, individual or corporate, possess money $m_{i}(t)$ at time $t$. In any trading, a pair of agents $i$ and $j$ randomly exchange their money $[7,8,9]$, such that their total money is (locally) conserved and none posses negative money $\left(m_{i}(t) \geq 0\right.$, i.e, debt not allowed):

$$
m_{i}(t+1)=m_{i}(t)+\Delta m ; m_{j}(t+1)=m_{j}(t)-\Delta m
$$




$$
\Delta m=\epsilon\left(m_{i}(t)+m_{j}(t)\right)-m_{i}(t) ; 0 \leq \epsilon \leq 1
$$

All the money transactions considered in this paper follow local conservation:

$$
m_{i}(t)+m_{j}(t)=m_{i}(t+1)+m_{j}(t+1) .
$$

The time $(t)$ changes by one unit after each trading and $\epsilon$ is a random fraction chosen independently for each trading or at each time $t$. The steady-state $(t \rightarrow \infty)$ distribution of money is Gibbs one:

$$
P(m)=(1 / T) \exp (-m / T) ; T=M / N .
$$

No matter how uniform or justified the initial distribution is, the eventual steady state corresponds to Gibbs distribution where most of the people have very little money. This follows from the conservation of money and additivity of entropy:

$$
P\left(m_{1}\right) P\left(m_{2}\right)=P\left(m_{1}+m_{2}\right) .
$$

This steady state result is quite robust and realistic. Several variations of the trading [1], does not affect the distribution.

In any trading, savings come naturally [19]. A saving factor $\lambda$ is therefore introduced in the same model [9] (Ref. 8] is the model without savings), where each trader at time $t$ saves a fraction $\lambda$ of its money $m_{i}(t)$ and trades randomly with the rest. In each of the following two cases, the savings fraction does not vary with time, and hence we call it 'quenched' in the terminology of statistical mechanics.

\section{B. Uniform savings}

For the case of 'uniform' savings, the money exchange rules remain the same (Eqn. (2)), where

$$
\Delta m=(1-\lambda)\left[\epsilon\left\{m_{i}(t)+m_{j}(t)\right\}-m_{i}(t)\right],
$$

where $\epsilon$ is a random fraction, coming from the stochastic nature of the trading. $\lambda$ is a fraction $(0 \leq \lambda<1)$ which we call the saving factor.

The market (non-interacting at $\lambda=0$ and 1) becomes effectively 'interacting' for any non-vanishing $\lambda(<1)$ : For uniform $\lambda$ (same for all agents), the steady state distribution $P_{f}(m)$ of money is sharply decaying on both sides with the most-probable money per agent shifting away from $m=0$ (for $\lambda=0$ ) to $M / N$ as $\lambda \rightarrow 1$. The self-organizing feature of this market, induced by sheer self-interest of saving by each agent without any global perspective, is very significant as the fraction of paupers decrease with saving fraction $\lambda$ and most people possess some fraction of the average money in the market (for $\lambda \rightarrow 1$, the socialists' dream is achieved with just people's self-interest of saving!). This uniform saving propensity does not give the Pareto-like power-law distribution yet, but the Markovian nature of the scattering or trading processes (eqn. (5)) is lost and the system becomes co-operative. Through $\lambda$, the agents indirectly get to develop a correlation with (start interacting with) each other and the system co-operatively self-organizes [20] towards a most-probable distribution.

This model has been understood to a certain extent (see e.g, [21, 22, 23]), and argued to resemble a gamma distribution [22], and partly explained analytically. This model clearly finds its relevance in cases where the economy consists of traders with 'waged' income [24].

\section{Distributed savings}

In a real society or economy, $\lambda$ is a very inhomogeneous parameter: the interest of saving varies from person to person. We move a step closer to the real situation where saving factor $\lambda$ is widely distributed within the population [11, 12, 13]. The evolution of money in such a trading can be written as:

$$
\begin{aligned}
m_{i}(t+1)= & \lambda_{i} m_{i}(t)+\epsilon \\
& \times\left[\left(1-\lambda_{i}\right) m_{i}(t)+\left(1-\lambda_{j}\right) m_{j}(t)\right], \\
m_{j}(t+1)= & \lambda_{j} m_{j}(t)+(1-\epsilon) \\
& \times\left[\left(1-\lambda_{i}\right) m_{i}(t)+\left(1-\lambda_{j}\right) m_{j}(t)\right]
\end{aligned}
$$

One again follows the same rules (Eqn. (2)) as before, except that

$$
\Delta m=\left(1-\lambda_{j}\right) \epsilon m_{j}(t)-\left(1-\lambda_{i}\right)(1-\epsilon) m_{i}(t)
$$

here; $\lambda_{i}$ and $\lambda_{j}$ being the saving propensities of agents $i$ and $j$. The agents have uniform (over time) saving propensities, distributed independently, randomly and uniformly (white) within an interval 0 to 1 agent $i$ saves a random fraction $\lambda_{i}\left(0 \leq \lambda_{i}<1\right)$ and this $\lambda_{i}$ value is quenched for each agent $\left(\lambda_{i}\right.$ are independent of trading or $t)$. $P(m)$ is found to follow a strict power-law decay. This decay fits to Pareto law (1) with $\nu=1.01 \pm 0.02$ for several decades. This power law is extremely robust: a distribution

$$
\rho(\lambda) \sim\left|\lambda_{0}-\lambda\right|^{\alpha}, \quad \lambda_{0} \neq 1, \quad 0 \leq \lambda<1,
$$

of quenched $\lambda$ values among the agents produce power law distributed $m$ with Pareto index $\nu=1$, irrespective of the value of $\alpha$. For negative $\alpha$ values, however, we get an initial (small $m$ ) Gibbs-like decay in $P(m)$. In case $\lambda_{0}=1$, the Pareto exponent is modified to $\nu=$ $1+\alpha$, which qualifies for the non-universal exponents in the same model [1], 25].

This model [11] has been thoroughly analyzed, and the analytical derivation of the Pareto exponent has been achieved in certain cases [23, 25, 26]. The Pareto exponent has been derived to exactly 1 .

In this model, agents with higher saving propensity tend to hold higher average wealth, which is justified by the fact that the saving propensity in the rich population is always high [28]. 


\section{IDEAL-GAS TRADING MARKET IN PRESENCE OF A NON-CONSUMABLE COMMODITY}

In the above markets, modifications due to exchange of a consumable commodity hardy affects the distribution, as the commodity once bought or sold need not be accounted for. Consumable commodities effectively have no 'price', as due to their short lifetime to contribute to the total wealth of an individual. It is interesting however, to study the role of non-consumable commodities in such market models and this we do here.

In the simplified version of a market with a single non-consumable commodity, we again consider a fixed number of traders or agents $N$ who trade in a market involving total money $\sum_{i} m_{i}(t)=M$ and total commodity $\sum_{i} c_{i}(t)=C, m_{i}(t)$ and $c_{i}(t)$ being the money and commodity of the $i$-th agent at time $t$ and are both non-negative. Needless to mention, both $m_{i}(t)$ and $c_{i}(t)$ change with time or trading $t$. The market, as before is closed, which means, $N, M$ and $C$ are constants. The wealth $w_{i}$ of an individual $i$ is thus, the sum of the money and commodity it possesses, i.e., $w_{i}=m_{i}+p_{0} c_{i} ; p_{0}$ is the "global" price. In course of trading, total money and total commodity are locally conserved, and hence the total wealth. In such a market, one can define a global average price parameter $p_{0}=M / C$, which is set here to unity, giving $w_{i}=m_{i}+c_{i}$. It may be noted here that in order to avoid the complication of restricting the commodity-money exchange and their reversal between the same agents, the Fisher velocity of money circulation (see e.g., Ref. [29]) is renormalised to unity here. In order to accommodate the lack of proper information and the ability of the agents to bargain etc., we will allow of course fluctuations $\delta$ in the price of the commodities at any trading (time): $p(t)=p_{0} \pm \delta=1 \pm \delta$. We find, the nature of steady state to be unchanged and independent of $\delta$, once it becomes nonvanishing.

\section{A. Dynamics}

In general, the dynamics of money in this market looks the same as Eqn. (2), with $\Delta m$ given by Eqns. (2), (5) or (8) depending on whether $\lambda_{i}=0$ for all, $\lambda_{i} \neq 0$ but uniform for all $i$ or $\lambda_{i} \neq \lambda_{j}$ respectively. However, all $\Delta m$ are not allowed here; only those, for which $\Delta m_{i} \equiv m_{i}(t+1)-m_{i}(t)$ or $\Delta m_{j}$ are allowed by the corresponding changes $\Delta c_{i}$ or $\Delta c_{j}$ in their respective commodities $(\Delta m>0, \Delta c>0)$ :

$$
\begin{aligned}
& c_{i}(t+1)=c_{i}(t)+\frac{m_{i}(t+1)-m_{i}(t)}{p(t)} \\
& c_{j}(t+1)=c_{j}(t)-\frac{m_{j}(t+1)-m_{j}(t)}{p(t)}
\end{aligned}
$$

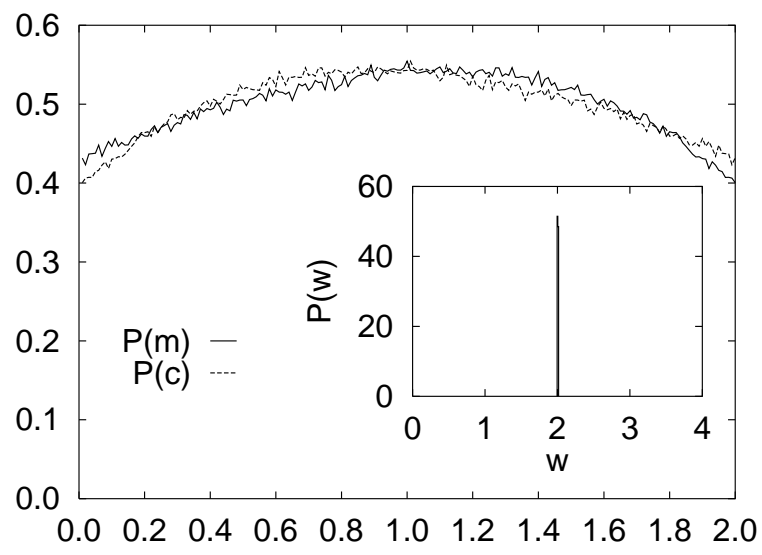

FIG. 1: Steady state distribution $P(m)$ of money $m$ in a market with no savings (saving factor $\lambda=0$ ) for no price fluctuations i.e, $\delta=0$. The graphs show simulation results for a system of $N=100$ agents, $M / N=1, C / N=1 ; m_{i}=1=c_{i}$ at $t=0$ for all agents $i$. The inset shows the distribution $P(w)$ of total wealth $w=m+c$. As $p=1$, for $\delta=0$, although $m$ and $c$ can change with tradings within the limit $(0-2)$ the sum is always maintained at 2 .

where $p(t)$ is the local-time 'price' parameter, a stochastic variable:

$$
p(t)= \begin{cases}1+\delta & \text { with probability } 0.5 \\ 1-\delta & \text { with probability } 0.5\end{cases}
$$

The role of the stochasticity in $p(t)$ is to imitate the effect of bargaining in a trading process. $\delta$ parametrizes the amount of stochasticity. The role of $\delta$ is significant in the sense that it determines the (relaxation) time the whole system takes to reach a dynamically equilibrium state; the system reaches equilibrium sooner for larger $\delta$, while its magnitude does not affect the steady state distribution. It may be noted that, in course of trading process, certain exchanges are not allowed (e.g., in cases when a particular pair of traders do not have enough commodity to exchange in favor of an agreed exchange of money). We then skip these steps and choose a new pair of agents for trading.

\section{B. Results}

For $\delta=0$, of course, the wealth of each agent remains invariant with time; only the proportion of money and commodity interchange within themselves, since the 'price' factor remains constant. This of course happens irrespective of the savings factor being zero, uniform or distributed. For $\delta=0$, the steady state distribution of money or commodity can take non-trivial forms: (see Fig. (1), but has strictly a $\delta$-function behavior for the total wealth distribution; it gets frozen at the value of wealth one starts with (see inset of Fig. 1 for the case $m_{i}=1=c_{i}$ for all $i$ ). 


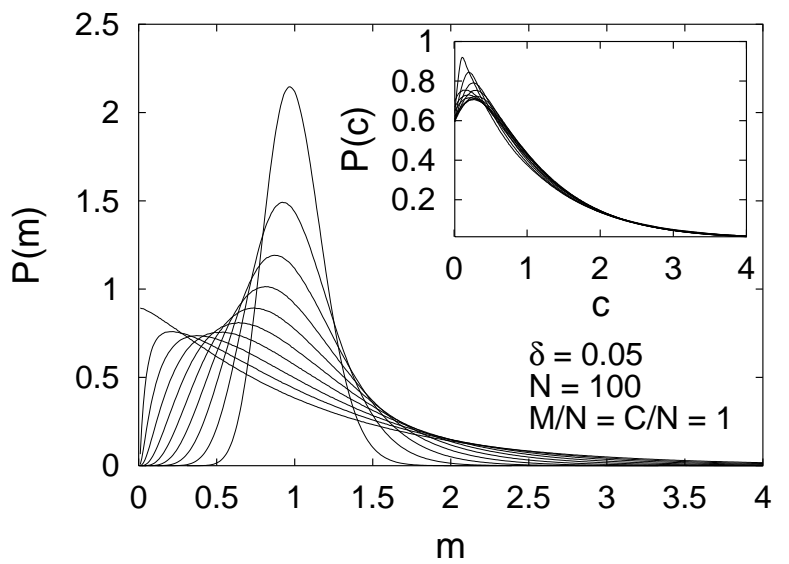

FIG. 2: Steady state distribution $P(m)$ of money $m$ in the uniform savings commodity market for different values of saving factor $\lambda(0,0.1,0.2,0.3,0.4,0.5,0.6,0.7,0.8,0.9$ from left to right near the origin) for $\delta=0.05$. The inset shows the distribution $P(c)$ of commodity $c$ in the uniform savings commodity market for different values of saving factor $\lambda$. The graphs show simulation results for a system of $N=100$ agents, $M / N=1, C / N=1$.

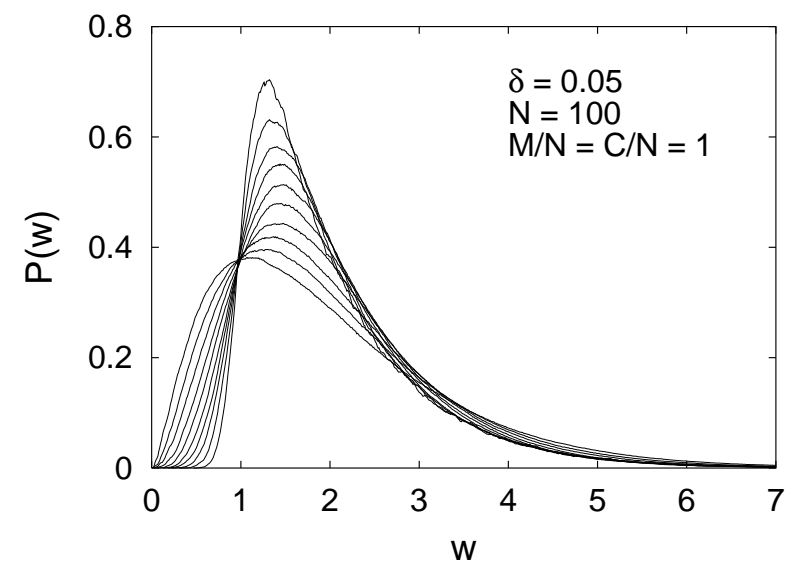

FIG. 3: Steady state distribution $P(w)$ of total wealth $w=m+c$ in the uniform savings commodity market for different values of saving factor $\lambda$ $(0,0.1,0.2,0.3,0.4,0.5,0.6,0.7,0.8,0.9$ from left to right) for $\delta=0.05$. The graphs show simulation results for a system of $N=100$ agents, $M / N=1, C / N=1$.

As mentioned already for $\delta \neq 0$, the steady state results are not dependent on the value of $\delta$ (the relaxation time of course decreases with increasing $\delta$ ). In such a market with uniform savings, money distribution $P(m)$ has a form similar to a set (for $\lambda \neq 0$ ) of Gamma functions (see Fig. 2): a set of curves with a most-probable value shifting from 0 to 1 as saving factor $\lambda$ changes from 0 to 1 (as in the case without any commodity). The commodity distribution $P(c)$ has an initial peak and an exponential fall-off, without much systematics with varying $\lambda$ (see inset of Fig. 22). The distribution $P(w)$ of total

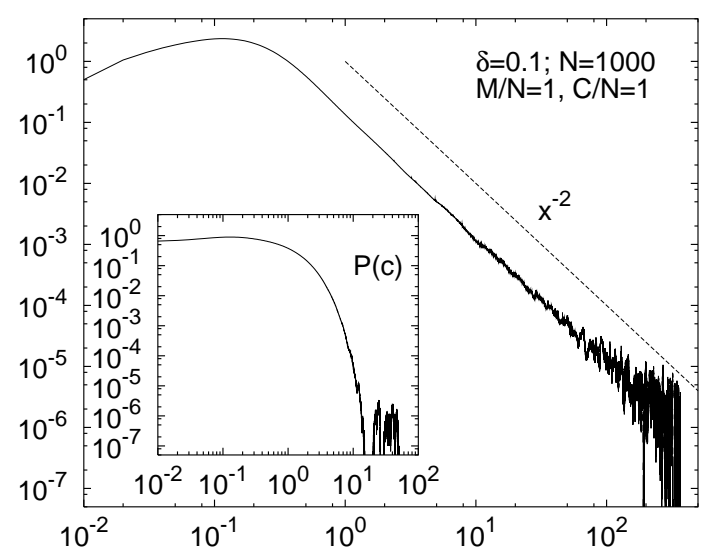

FIG. 4: Steady state distribution $P(m)$ of money $m$ in the commodity market with distributed savings $0 \leq \lambda<1 . P(m)$ has a power-law tail with Pareto index $\nu=1 \pm 0.02$ (a power law function $x^{-2}$ is given for comparison). The inset shows the distribution $P(c)$ of commodity $c$ in the same commodity market. The graphs show simulation results for a system of $N=1000$ agents, $M / N=1, C / N=1$.

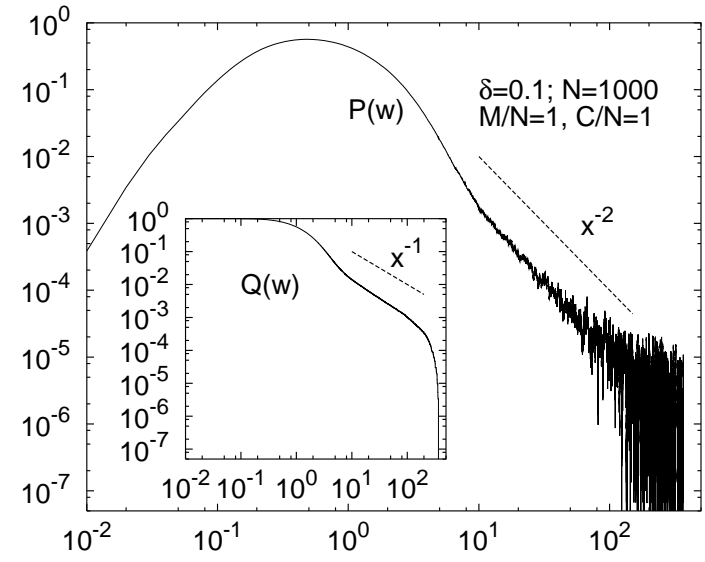

FIG. 5: Steady state distribution $P(w)$ of total wealth $w=$ $m+c$ in the commodity market with distributed savings $0 \leq$ $\lambda<1$. $P(w)$ has a power-law tail with Pareto index $\nu=1 \pm$ 0.05 (a power law function $x^{-1}$ is given for comparison). The inset shows the cumulative distribution $Q(w) \equiv \int_{w}^{\infty} P(w) \mathrm{d} w$. The graphs show simulation results for a system of $N=1000$ agents, $M / N=1, C / N=1$.

wealth $w=m+c$ behaves much like $P(m)$ (see Fig. 3). It is to be noted that since there is no precise correspondence with commodity and money for $\delta \neq 0$ (unlike when $\delta=0$, when the sum is fixed), $P(w)$ cannot be derived directly from $P(m)$ and $P(c)$. However, there are further interesting features. Although they form a class of Gamma distributions, the set of curves for different values of saving factor $\lambda$ seem to intersect at a common point, near $w=1$. All the reported data are for a system of $N=100$ agents, with $M / N=1$ and $C / N=1$ and for a case where the noise level $\delta$ equals $10 \%$. 
For $\lambda$ distributed uniformly within the interval $0 \leq$ $\lambda<1$, the tails of both money and wealth distributions $P(m)$ and $P(w)$ have Pareto law behavior with a fitting exponent value $\nu=1 \pm 0.02$ and $\nu=1 \pm 0.05$ respectively (see Figs. 4 and 5 respectively), whereas the commodity distribution is still exponentially decaying (see inset of Fig. 4).

\section{SUMMARY AND CONCLUSIONS}

Let us first summarize the results for the models without any commodity (money-only exchange models): There are $N$ players participating in a game, each having an initial capital of one unit of money. $N$ is very large, and total money $M=N$ remains fixed over the game (so also the number of players $N$ ). (a) In the simplest version, the only move at any time is that two of these players are randomly chosen and they decide to share their total money randomly among them. As one can easily guess, the initial uniform (a delta function) distribution of money will soon disappear. Let us ask what the eventual steady state distribution of money after many such moves will be? At each move, a pair of randomly chosen players share a random fraction of their total money among themselves. The answer is well established in physics for more than a century - soon, there will be a stable money distribution and it will be Gibbs distribution: $P(m) \sim \exp [-m / T] ; T=M / N$ [8]. (b) Now think of a modified move in this game: each player 'saves' a fraction $\lambda$ of the money available with him/her after each move and while going to the next move. Everybody saves the same fraction $\lambda$. What is the steady state distribution of money after a large number of such moves? It becomes Gamma-function like, while the distribution parameters of course depend on $\lambda$ (see Ref. [9, 22]) see also Ref. [17, 18]; for a somewhat different model with similar results developed much earlier. No exact treatment of this problem is available so far. (c) What happens to the eventual money distribution among these players if $\lambda$ is not the same for all players but is different for different players? Let the distribution $\rho(\lambda)$ of saving propensity $\lambda$ be such that $\rho(\lambda)$ is non-vanishing when $\lambda \rightarrow 1$. The actual distribution will depend on the saving propensity distribution $\rho(\lambda)$, but for all of them, the asymptotic form of the distribution will become Paretolike: $P(m) \sim m^{-\alpha} ; \alpha=2$ for $m \rightarrow \infty$. This is valid for all such distributions (unless $\rho(\lambda) \propto(1-\lambda)^{\beta}$, when $\left.P(m) \sim m^{-(2+\beta)}\right)$. However, for variation of $\rho(\lambda)$ such that $\rho(\lambda) \rightarrow 0$ for $\lambda<\lambda_{0}$, one will get an initial Gamma function form for $P(m)$ for small and intermediate values of $m$, with parameters determined by $\lambda_{0}(\neq 0)$, and this distribution will eventually become Pareto-like for $m \rightarrow \infty$ [11, 12, 23]. A somewhat rigorous analytical treatment of this problem is now available [25].

A major limitation of these money-only exchange models considered earlier 1, 6, 7, 8, 9, 10, 11, 12, 13, 14, 15, 16, 17, 18, 22, 23, 25, 26, 27] (and summarised in (a), (b) and (c) above) is that it does not make any explicit reference to the commodities exchanged with the money and to the constraints they impose. Also, the wealth is not just the money is possession (unless the commodity exchanged with the money is strictly consumable). Here, we study the effect of a single non-consumable commodity on the money (and also wealth) distributions in the steady state, allowing for the total (in time) price fluctuation. This allowance of price fluctuation here is very crucial for the model; it allows for the stochastic dynamics to play its proper role in the market. As such, this model is therefore quite different from that considered recently in Ref. [30], where $p_{0}$ is strictly unity and the stochasticity enters from other exogenous factors. In the sense that we also consider two exchangeable variables in the market, our model has some similarity with that in Ref [31]. However, Silver et al [31] consider only random exchanges between them (keeping the total conserved) while we consider random exchanges permitting price fluctuations and savings. As such they get only the Gamma distribution in wealth, while we get both Gamma and Pareto distributions.

In spite of many significant effects due to the inclusion of a non-consumable commodity, the general feature of Gamma-like form of the money (and wealth) distributions (for uniform $\lambda$ ) and the power law tails for both money and wealth (for distributed $\lambda$ ) with identical exponents, are seen to remain unchanged. The precise studies (theories) for the money-only exchange models are therefore extremely useful and relevant.

Specifically, we study here numerically the behavior of an ideal gas like model of markets having only one nonconsumable commodity. The total amount of money in the market $M=\sum_{i} m_{i}, i=1, \ldots, N$ is fixed, so is the total amount of commodity $C=\sum_{i} c_{i}$ and of course the total number of agents $N$ in the market. As in the market there is only one commodity, which is nonconsumable, we normalize its global price $p_{0}=M / C$ to unity. The wealth of any agent $i$ at any time $t$ is therefore $w_{i}(t)=m_{i}(t)+c_{i}(t)$. If no fluctuation in price $p$ is allowed (over $p_{0}$ ), then the money-commodity exchange leads to trivial money and commodity distribution as shown in Fig. 1, which keeps the wealth of any agent unchanged over time. If we now allow the price $p(t)$ at any time to fluctuate over $p_{0}$ by a factor $\delta$ (as in (12)), nontrivial money, commodity and wealth distributions set in (the steady states of which are independent of $\delta ; \delta \neq 0$ ). We investigated here the behavior of the steady-state distributions of money, commodity and total wealth, as this dynamics of trading or exchange of money or commodity proceeds, allowing for temporal fluctuations in the price of the commodity. These distributions are studied in markets with agents having uniform (see Figs. 2 and 3) and random saving factors (see Figs. 4 and 5). The self-organizing features in money distribution are similar to the cases without any commodity (or with consumable commodities), the commodity distribution shows an exponential decay. The wealth distribution shows interest- 
ing behavior: Gamma like distribution for uniform saving propensity and has a power-law tail (with Pareto exponent value $\nu=1$ ) for a market with agents having random saving propensity. Although our results are numerical, and the Pareto behavior for the wealth distribution tail gets somewhat more restricted in range (compared to that of the money distribution; see Fig. (4)), the ro- bustness of the power-law behavior nevertheless becomes obvious from, say Fig. (4), where the power law tail for the money distribution clearly dominates over the commodity distribution tail, which rapidly decays off exponentially.

We are extremely grateful to Anindya-Sundar Chakrabarti for useful suggestions and comments.
[1] Econophysics of Wealth Distributions, Eds. A. Chatterjee, S. Yarlagadda, B. K. Chakrabarti, Springer Verlag, Milan (2005)

[2] V. Pareto, Cours d'economie Politique, F. Rouge, Lausanne and Paris (1897)

[3] M. Levy, S. Solomon, Physica A 24290 (1997); A. A. Drăgulescu, V. M. Yakovenko, Physica A 299213 (2001); H. Aoyama, W. Souma, Y. Fujiwara, Physica A 324352 (2003)

[4] A. Banerjee, V. M. Yakovenko, T. Di Matteo, Physica A 37054 (2006); F. Clementi, M. Gallegati, Physica A 350 427 (2005)

[5] S. Sinha, Physica A 359555 (2006)

[6] S. Sinha, Phys. Scripta T 10659 (2003); J. C. Ferrero, Physica A 341575 (2004); J. R. Iglesias, S. Gonçalves, G. Abramson, J. L. Vega, Physica A 342186 (2004); N. Scafetta, S. Picozzi, B. J. West, Physica D 193338 (2004)

[7] B. K. Chakrabarti, S. Marjit, Indian J. Phys. B 69681 (1995); S. Ispolatov, P. L. Krapivsky, S. Redner, Eur. Phys. J. B 2267 (1998)

[8] A. A. Drăgulescu, V. M. Yakovenko, Eur. Phys. J. B 17 $723(2000)$

[9] A. Chakraborti, B. K. Chakrabarti, Eur. Phys. J. B 17 167 (2000)

[10] B. Hayes, American Scientist, USA 90 (Sept-Oct) 400 (2002)

[11] A. Chatterjee, B. K. Chakrabarti, S. S. Manna, Physica A 335155 (2004)

[12] A. Chatterjee, B. K. Chakrabarti, S. S. Manna, Physica Scripta T 10636 (2003)

[13] B. K. Chakrabarti, A. Chatterjee, in Application of Econophysics, Ed. H. Takayasu, Springer, Tokyo (2004) pp. $280-285$
[14] F. Slanina, Phys. Rev. E 69046102 (2004)

[15] A. KarGupta, in Econophysics and Sociophysics: Trends and Perspectives, Eds. B. K. Chakrabarti, A. Chakraborti, A. Chatterjee, Wiley-VCH, Berlin, pp 161190 (2006); P. Richmond et al, ibid pp. 131-159 (2006)

[16] A. Chatterjee and B. K. Chakrabarti in [1]

[17] J. Angle, Social Forces 65293 (1986)

[18] J. Angle, Physica A 367388 (2006)

[19] P. A. Samuelson, Economics, Mc-Graw Hill Int., Auckland (1980)

[20] P. Bak, How Nature works, Oxford University Press, Oxford (1997)

[21] A. Das, S. Yarlagadda, Phys. Scripta T 10639 (2003)

[22] M. Patriarca, A. Chakraborti, K. Kaski, Phys. Rev. E 70016104 (2004)

[23] P. Repetowicz, S. Hutzler, P. Richmond, Physica A 356 641 (2005)

[24] J. Mimkes, G. Willis in [1]

[25] P. K. Mohanty, Phys. Rev. E 74011117 (2006)

[26] A. Chatterjee, B. K. Chakrabarti, R. B. Stinchcombe, Phys. Rev. E 72026126 (2005)

[27] A. Chatterjee, B. K. Chakrabarti, xxx.arxiv.org/physics/0607258

[28] K. E. Dynan, J. Skinner, S. P. Zeldes, J. Pol. Econ. 112 397 (2004)

[29] Y. Wang, N. Xi, N. Ding in Econophysics and Sociophysics: Trends and Perspectives, Eds. B. K. Chakrabarti, A. Chakraborti, A. Chatterjee, WileyVCH, Berlin, pp 191-217 (2006)

[30] M. Ausloos, A. Peķalski, Physica A 373560 (2007)

[31] J. Silver, E. Slud, K. Takamoto, J. Econ. Theory 106 417 (2002). 